\title{
O cuidado com a população em situação de rua: o consultório na rua nas Redes de Atenção à Saúde e Intersetorial: revisão integrativa da literatura
}

\section{Health care for population in street situation: the street outreach offices in the health and intersectorial network}

\author{
Paulo Alexandre Françoso $^{1}$, Elisabete Ferreira Mângia ${ }^{2}$, Melissa Tieko Muramoto ${ }^{3}$ \\ http://dx.doi.org/10.11606/issn.2238-6149.v30i2p124-131
}

Françoso PA, Mângia EF, Muramoto MT. O cuidado com a população em situação de rua: o consultório na rua nas Redes de Atenção à Saúde e Intersetorial: revisão integrativa da literatura. Rev Ter Ocup Univ São Paulo. 2020 maio-ago.;30(2):124-31.

RESUMO: O Consultório na Rua $(\mathrm{CnR})$ nasce como dispositivo que visa garantir acesso a cuidados em saúde para a População em Situação de Rua (PSR), tendo como uma de suas atribuições a articulação da Rede de Saúde e Intersetorial, em reconhecimento à multiplicidade de necessidades dessa população e a partir de concepção ampliada de saúde. Há, no entanto, desafios a serem superados para a efetivação desse trabalho, que variam desde burocracias que necessitam ser flexibilizadas para se adequar à realidade da PSR, até o preconceito e as contradições entre ações dos diversos atores estatais que se relacionam com essa população. O CnR tem sido potente no enfrentamento desse cenário, mas também insuficiente frente à profundidade das estruturas sociais e econômicas que o sustentam.

DESCRITORES: Visitas a consultório médico; Serviços de saúde; Pessoas em situação de rua; Redes comunitárias; Colaboração intersetorial.
Françoso PA, Mângia EF, Muramoto MT. Health care for population in street situation: the street outreach offices in the health and intersectorial network. Rev Ter Ocup Univ São Paulo. 2020 May-Aug.;30(2):124-31.

ABSTRACT: The Street Outreach Office (SOO) is born as a device that aims to guarantee access to health care for the Population in Street Situation (PSS). One of its attributions is the articulation of the Health and Intersectorial Network, in recognition of the multiplicity of needs of this population, based in an expanded conception of health. However, there are challenges to be overcome for the effective work in this area, ranging from bureaucracies that need to be flexibilized to fit the reality of PSS, to the prejudgement, and contradictions between actions of the various state actors that relate to this population. The SOO has been potent in facing this scenario, but also insufficient in the depth of the social and economic structures that sustain it.

KEYWORDS: Office visits; Health services; Homeless persons; Community networks; Intersectorial collaboration.

Este trabalho foi apresentado como trabalho de conclusão de curso no Programa de Residência Multiprofissional em Saúde Mental com Ênfase em Dependência Química da Faculdade de Medicina da Universidade de São Paulo.

1. Psicólogo na ABECAL - Associação Beneficente Caminho de Luz. https://orcid.org/0000-0001-5271-7802. Email: paulotecweb@gmail.com

2. Professora Associada Livre Docente, Faculdade de Medicina da Universidade de São Paulo. https://orcid.org/0000-0002-2572-6639. Email: mangeli@usp.br.

3. Terapeuta Ocupacional, Faculdade de Medicina da Universidade de São Paulo. https://orcid.org/0000-0002-7868-6300. Email: melissatieko@usp.br.

Endereço para correspondência: Paulo A. Françoso. Email: paulotecweb@gmail.com. 


\section{INTRODUÇÃO}

$\mathrm{O}$ acesso universal à saúde é direito garantido pela constituição brasileira, contudo, ainda existem populações em situação de vulnerabilidade, como é o caso da população em situação de rua que vivenciam barreiras no acesso aos cuidados em saúde ${ }^{1}$.

Para o enfrentamento dessa problemática, foi criado o primeiro Consultório de Rua na Bahia, em 1995, vinculado ao Centro de Estudos e Terapia do Abuso de Drogas, com foco nos jovens usuários de substâncias psicoativas². Em 2004, inspirado na experiência do CETAD, foi implantado um CR junto ao primeiro Centro de Atenção Psicossocial Álcool e outras Drogas (CAPS-AD) de Salvador ${ }^{3}$.

Reconhecido, em 2009, como parte do Plano Emergencial de Ampliação de Acesso ao Tratamento e Prevenção em Álcool e outras Drogas ${ }^{4}$, somente em 2011, sob a nova nomenclatura Consultório na Rua (CnR), esse dispositivo foi realinhado de forma a integrar-se à Atenção Básica em Saúde - ABS $^{5}$.

A integração do $\mathrm{CnR}$ na $\mathrm{ABS}$, além de estar consonante com a variedade de necessidades em saúde da PSR, também confere a esse dispositivo nova atribuição, uma vez que o Sistema Único de Saúde (SUS) se organiza como Rede de Atenção à Saúde - $\mathrm{RAS}^{3}$ na qual a $\mathrm{ABS}$ recebe a função de articular os demais pontos da RAS, conforme as necessidades de saúde dos usuários do sistema ${ }^{5}$. Além disso, é prevista a responsabilidade do $\mathrm{CnR}$ na articulação da rede intersetorial ${ }^{6}$, reconhecendo a multiplicidade de necessidades da PSR e operando segundo concepção ampliada de saúde. Importa ressaltar que, a partir do rearranjo das equipes de Consultório na Rua (eCnR) como agentes da ABS, seu escopo de atuação não foi alterado, mas ampliado, uma vez que o $\mathrm{CnR}$ passou a figurar simultaneamente como ponto de atenção da ABS e da Rede de Atenção Psicossoal - RAPS ${ }^{7}$. Desse modo, além de articular as redes, o CnR é também responsável por ações no âmbito individual e coletivo em promoção, proteção e manutenção da saúde, prevenção de agravos, diagnóstico, tratamento, reabilitação e redução de danos ${ }^{5}$.

Apesar do trabalho em rede constituir diretriz oficial do SUS, existem desafios implicados na prática cotidiana que foram alvo de estudos e experiências brasileiras anteriores à edição das atuais diretrizes do SUS $8,9,10,11$. Desses estudos, foram originadas as noções de Equipe de Referência e Apoio Matricial que mais tarde foram incorporadas na Política de Saúde ${ }^{3,5,7}$, conferindo fundamento teórico à organização e articulação de rede, com vistas a superar as formas tradicionais de relação entre serviços atravessadas pela burocracia e desequilíbrio de poder ${ }^{12}$.
Em suma, a Equipe de Referência é um arranjo no qual os profissionais de uma equipe assumem o acompanhamento de saúde de determinada clientela com coordenação interdisciplinar ${ }^{12}$. Isso se dá em diversos níveis, podendo, em situações concretas, a clientela significar um indivíduo, uma família, uma comunidade ou um território ${ }^{9,11}$.

A Equipe de Referência tem por objetivo garantir a construção do vínculo, a relação terapêutica longitudinal e o acompanhamento da atenção à saúde de cada usuário rompendo com o poder gerencial fragmentado pela centralidade em procedimentos e profissões ${ }^{9,11,13}$. Pressupõe a interdependência entre saberes profissionais, possibilitando o olhar interdisciplinar sobre o usuário e suas necessidades de saúde ${ }^{12}$.

A Equipe de Referência se articula com profissionais e equipes que não participam de seu cotidiano de trabalho em busca de suporte técnico especializado. A esses profissionais e equipes é atribuída a função de Apoio Matricial, que pode ser prestado tanto sob a forma de retaguarda assistencial, objetivando o cuidado direto e compartilhado dos usuários, quanto na modalidade de suporte técnico-pedagógico, visando o aumento da capacidade resolutiva da Equipe de Referência ${ }^{12,13}$.

Colocar em prática o trabalho em rede, pautado nos arranjos organizativos da Equipe de Referência e do Apoio Matricial, significa enfrentar obstáculos oriundos da compreensão tradicional do trabalho em saúde, conforme apontam Campos e Domitti ${ }^{11}$ : estrutura organizacional fragmentada, carência de recursos, concentração de poder, cultura de concorrência, epistemologia restrita e questões éticas.

Partindo dessa base teórica e normativa, este trabalho tem o objetivo de identificar os desafios e potências enfrentados no cotidiano do trabalho, no contexto da articulação de redes envolvendo o $\mathrm{CnR}$.

\section{MÉTODO}

Trata-se de estudo na modalidade de revisão integrativa da literatura sobre o trabalho de articulação de rede de saúde e intersetorial na atuação do CnR. A revisão integrativa é uma metodologia que visa sintetizar o conhecimento para incorporar na prática os resultados de estudos significativos ${ }^{14}$.

A questão de pesquisa que guiou o levantamento dos estudos foi construída com base na estratégia SPIDER. A estratégia SPIDER guia a pesquisa pelos elementos constitutivos de sua sigla: Sample (S), Phenomenon of Interest (PI), Design (D), Evaluation (E) e Research Type (R) ${ }^{15}$.

Desse modo, o levantamento dos estudos foi realizado entre junho e julho de 2017 a partir da questão norteadora 
"Como ocorre (E) o trabalho de articulação de Rede de Saúde e de Rede Intersetorial (PI) no cotidiano do serviço CnR (S)".

Assim, tendo o objetivo definido (PI/E), foram definidos os critérios de inclusão para a busca: estudos qualitativos com uso de qualquer metodologia (D/R), que tenham por participantes trabalhadores e/ou usuários de serviços de CnR (S), publicados após 2011 - ano em que entra em vigor a integração do $\mathrm{CnR}$ na $\mathrm{ABS}(\mathrm{S})$. As bases de dados consultadas foram SciELO, BVS Saúde e Scopus, fontes que não fazem uso de vocabulário controlado.

Considerando a ausência de literatura focada no objeto dessa revisão, optou-se por pesquisar a palavra-chave "Consultório na Rua" para levantar estudos variados sobre o trabalho desse serviço $(\mathrm{S})$ e, por meio de análise posterior, filtrar os estudos que respondem ao objetivo dessa revisão (PI/E).

\begin{tabular}{|c|c|c|c|}
\hline \multirow{3}{*}{ } & $\operatorname{SciELO}(\mathrm{n}=18)$ & BVS Saúde $(\mathrm{n}=19)$ & Scopus $(n=12)$ \\
\hline & \multicolumn{3}{|c|}{$\begin{array}{l}\text { Registros sem texto completo disponível e removidos }(\mathrm{n}=2) \\
\text { Registros duplicados e removidos }(\mathrm{n}=24)\end{array}$} \\
\hline & Scielo $(n=11)$ & BVS Saúde $(\mathrm{n}=5)$ & Scopus $(n=7)$ \\
\hline \multicolumn{4}{|c|}{ Total: 23} \\
\hline
\end{tabular}

\begin{tabular}{|c|c|c|}
\hline 热 & $\begin{array}{c}\text { Excluídos na avaliação de títulos } \\
\text { e resumos }(\mathrm{n}=12)\end{array}$ & $\begin{array}{c}\text { Motivos: carta; estudo com temática diferente do objeto dessa pesquisa; } \\
\text { exploração teórica ou documental sem dados de práticas; estudo em } \\
\text { contexto estrangeiro; estudo não focado no trabalho do consultório na rua. }\end{array}$ \\
\hline \multicolumn{2}{|c|}{ Total: 11} \\
\hline
\end{tabular}

\begin{tabular}{|c|c|c|}
\hline \multirow{2}{*}{ 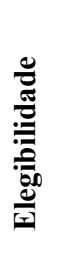 } & \multicolumn{2}{|c|}{$\begin{array}{c}\text { Registros recuperados }(n=11) \\
\text { Scielo }(n=8)-\text { BVS Saúde }(n=3)-\text { Scopus }(n=0)\end{array}$} \\
\hline & Excluídos na leitura do texto completo $(n=4)$ & $\begin{array}{l}\text { Motivos: estudo sem foco no trabalho do } \\
\text { consultório na rua; estudo sem dados de práticas } \\
\text { de trabalho em rede; estudo com temática } \\
\text { diferente do objeto dessa pesquisa. }\end{array}$ \\
\hline
\end{tabular}

\begin{tabular}{|c|c|}
\hline $\begin{array}{l}\stackrel{\mathscr{O}}{O} \\
\stackrel{\Xi}{\Xi}\end{array}$ & $\begin{array}{c}\text { Amostra Final }(\mathrm{n}=7) \\
\text { Scielo }(\mathrm{n}=5)-\text { BVS Saúde }(\mathrm{n}=2)-\text { Scopus }(\mathrm{n}=0)\end{array}$ \\
\hline
\end{tabular}

Figura 1 - Fluxograma do processo de construção da amostragem de revisão

Após levantamento de amostragem inicial, foi realizada nova seleção a partir da leitura de títulos e resumos em busca de estudos que trouxessem dados de práticas de eCnR.

Por último foi realizada a leitura integral dos textos em busca de pesquisas que trouxessem dados do trabalho de eCnR relativo a articulação de Rede de Saúde e/ou Intersetorial, que constitui objeto desta revisão.

Para a análise dos artigos, optou-se pela construção da matriz de síntese, ancorada na pergunta da pesquisa, e os resultados são apresentados de forma descritiva ${ }^{15}$.

\section{RESULTADOS}

A amostragem final foi composta por 7 artigos. Esses trabalhos foram publicados entre 2014 e 2016, tendo sido realizadas as coletas de dados entre 2010 e 2015 , fornecendo diferentes panoramas da atuação das eCnR considerando a integração desse dispositivo na ABS em 2011. Um dos estudos ${ }^{16}$ não informou o período de coleta de dados, tendo obtido aprovação do comitê de ética em pesquisa em 2011. 
Os dados relativos à articulação de Rede de Saúde e Intersetorial promovida pelas eCnR foram categorizados em dois grandes grupos respectivamente denominados "desafios", compreendendo obstáculos a serem superados, e "potências", indicando pontos fortes da atuação de eCnR. Estes dados estão sumarizados na Figura 2.

\begin{tabular}{|c|c|c|c|c|}
\hline $\begin{array}{l}\text { Autores } \\
\text { (ano) }\end{array}$ & $\begin{array}{l}\text { Tipo de } \\
\text { estudo }(D / R)\end{array}$ & Participantes (S) & \multicolumn{2}{|c|}{ Trabalho em Rede (PI) } \\
\hline \multirow{2}{*}{$\begin{array}{l}\text { Souza et al. } \\
(2014)\end{array}$} & \multirow{2}{*}{$\begin{array}{l}\text { Pesquisa } \\
\text { qualitativa; } \\
\text { Estudo } \\
\text { exploratório e } \\
\text { descritivo }\end{array}$} & \multirow{2}{*}{$\begin{array}{l}\text { Trabalhadores de uma } \\
\text { eCnR vinculada a um } \\
\text { CAPS AD da região } \\
\text { metropolitana de Recife }\end{array}$} & Desafios & $\begin{array}{l}\text {-Barreiras burocráticas } \\
\text {-Preconceito } \\
\text {-Carência de serviços } \\
\end{array}$ \\
\hline & & & Potências & $\begin{array}{l}\text {-Reconhecimento da necessidade de articular redes de } \\
\text { saúde e intersetorial } \\
\text {-Aprofundamento sobre o significado de trabalho em rede } \\
\text { - CnR como promotor do acesso }\end{array}$ \\
\hline \multirow[t]{2}{*}{$\begin{array}{l}\text { Hallais e } \\
\text { Barros (2015) }\end{array}$} & \multirow{2}{*}{$\begin{array}{l}\text { Pesquisa } \\
\text { qualitativa; } \\
\text { Observação } \\
\text { participante }\end{array}$} & \multirow[t]{2}{*}{$\begin{array}{l}\text { Trabalhadores de uma } \\
\text { eCnR de Campinas, São } \\
\text { Paulo }\end{array}$} & Desafios & $\begin{array}{l}\text {-Barreiras Burocráticas } \\
\text {-Preconceito } \\
\text {-Contradições entre políticas públicas }\end{array}$ \\
\hline & & & Potências & - CnR como promotor do acesso \\
\hline \multirow{2}{*}{$\begin{array}{l}\text { Lima e Seidl } \\
(2015)\end{array}$} & \multirow{2}{*}{$\begin{array}{l}\text { Pesquisa } \\
\text { qualitativa; } \\
\text { Estudo } \\
\text { descritivo e } \\
\text { exploratório }\end{array}$} & \multirow{2}{*}{$\begin{array}{l}\text { Trabalhadores de uma } \\
\text { eCnR de Goiânia e usuários } \\
\text { atendidos pela equipe } \\
\text { pesquisada }\end{array}$} & Desafios & $\begin{array}{l}\text {-Barreiras Burocráticas } \\
\text {-Preconceito } \\
\text {-Fragilidades na articulação intersetorial } \\
\end{array}$ \\
\hline & & & Potências & $\begin{array}{l}\text {-Reconhecimento da necessidade de articular redes de } \\
\text { saúde e intersetorial } \\
\text { - CnR como promotor do acesso }\end{array}$ \\
\hline \multirow[t]{2}{*}{$\begin{array}{l}\text { Silva et al. } \\
(2015)\end{array}$} & \multirow[t]{2}{*}{$\begin{array}{l}\text { Pesquisa } \\
\text { qualitativa; } \\
\text { Estudo de Caso }\end{array}$} & \multirow{2}{*}{$\begin{array}{l}\text { Profissionais de saúde que } \\
\text { atuam diretamente com a } \\
\text { PSR (maioria de eCnR); } \\
\text { profissionais de espaço } \\
\text { de educação permanente; } \\
\text { e um representante de } \\
\text { movimento social. }\end{array}$} & Desafios & $\begin{array}{l}\text {-Barreiras Burocráticas } \\
\text {-Preconceito } \\
\text {-Carência de serviços } \\
\text {-Contradições entre políticas públicas } \\
\text {-Necessidades formativas } \\
\text {-Dinâmica de viver na rua }\end{array}$ \\
\hline & & & Potências & $\begin{array}{l}\text {-Reconhecimento da necessidade de articular redes de } \\
\text { saúde e intersetorial } \\
\text {-Aprofundamento sobre o significado de trabalho em rede } \\
\text { - CnR como promotor do acesso }\end{array}$ \\
\hline \multirow[b]{2}{*}{$\begin{array}{l}\text { Engstron } \\
\text { e Teixeira } \\
(2016)\end{array}$} & \multirow{2}{*}{$\begin{array}{l}\text { Pesquisa } \\
\text { qualitativa; } \\
\text { Relato de } \\
\text { experiência }\end{array}$} & \multirow[b]{2}{*}{$\begin{array}{l}\text { eCnR de Manguinhos, Rio } \\
\text { de Janeiro }\end{array}$} & Desafios & $\begin{array}{l}\text {-Carência de serviços } \\
\text { - Necessidades formativas }\end{array}$ \\
\hline & & & Potências & $\begin{array}{l}\text {-Reconhecimento da necessidade de articular redes de } \\
\text { saúde e intersetorial } \\
\text {-Aprofundamento sobre o significado de trabalho em rede } \\
\text { - CnR como promotor do acesso }\end{array}$ \\
\hline \multirow{2}{*}{$\begin{array}{l}\text { Ferreira et al. } \\
(2016)\end{array}$} & \multirow{2}{*}{$\begin{array}{l}\text { Pesquisa } \\
\text { qualitativa; } \\
\text { participativa }\end{array}$} & \multirow{2}{*}{$\begin{array}{l}\text { PSR atendida por equipes } \\
\text { de } C n R \text { de Maceió }\end{array}$} & Desafios & $\begin{array}{l}\text {-Barreiras Burocráticas } \\
\text {-Preconceito } \\
\text {-Fragilidades na articulação intersetorial }\end{array}$ \\
\hline & & & Potências & $\begin{array}{l}\text {-Reconhecimento da necessidade de articular redes de } \\
\text { saúde e intersetorial } \\
\text { - CnR como promotor do acesso }\end{array}$ \\
\hline \multirow[b]{2}{*}{$\begin{array}{l}\text { Kami et al } \\
(2016)\end{array}$} & \multirow[b]{2}{*}{$\begin{array}{l}\text { Pesquisa } \\
\text { qualitativa; } \\
\text { exploratória }\end{array}$} & \multirow[b]{2}{*}{$\begin{array}{l}\text { Trabalhadores de um CnR } \\
\text { de Curitiba e usuários } \\
\text { atendidos pelo serviço }\end{array}$} & Desafios & - Necessidades formativas \\
\hline & & & Potências & $\begin{array}{l}\text {-Reconhecimento da necessidade de articular redes de } \\
\text { saúde e intersetorial } \\
\text { - CnR como promotor do acesso }\end{array}$ \\
\hline
\end{tabular}

Figura 2 - Síntese dos achados orientado pela estratégia SPIDER. 


\section{DESAFIOS}

Os desafios mais apontados nos estudos foram as barreiras burocráticas e o preconceito dos profissionais que integram os serviços da rede, tendo ambos aparecido em cinco estudos.

As barreiras burocráticas aparecem como dificultadores do acesso aos serviços por meio da exigência de documentação para cadastramento nas unidades, as quais, frequentemente, a PSR não possui ${ }^{16,17,18,19,20}$. Essa problemática também é mencionada em um dos estudos quanto à necessidade de dispensação de medicamentos antirretrovirais, pois estes só podem ser obtidos mediante a cadastro com apresentação de documento original com foto ${ }^{20}$. Houve também menção de entraves de gestão na garantia de espaço físico para as eCnR dentro das UBS. A inserção das eCnR no espaço físico de UBS é uma estratégia para ampliação do reconhecimento do direito de acesso da PSR aos cuidados da $\mathrm{ABS}^{19}$.

O preconceito também é apontado como fator limitador do acesso da PSR ${ }^{16,17,18,19}$. As bases desse preconceito variam entre a própria situação de rua, o uso de substâncias psicoativas (SPA), as condições de higiene e dos cuidados com a saúde. Hallais e Barro ${ }^{18}$ apontam para a relação estrutural entre as disparidades socioeconômicas e as condições desiguais de acesso aos serviços de saúde, sendo que no caso da PSR isso se reflete em processos de exclusão e invisibilização de seus direitos.

A carência de serviços específicos para o atendimento das necessidades da PSR nos territórios estudados foi evidenciada em três estudos. Souza et al. ${ }^{17}$ aponta para a necessidade de serviços que componham uma rede assistencial noturna. Silva et al. ${ }^{21}$ denuncia a ausência de CAPS - AD. Engstron e Teixeira ${ }^{1}$, por sua vez, indicam a escassez de unidades de acolhimento e a inexistência de serviços de atenção psicossocial em regime residencial.

As contradições entre políticas públicas foram abordadas em dois estudos. Elas se expressam em ações que ora violentam e segregam e ora se propõe a cuidar e incluir ${ }^{21}$. Nesse sentido, observou-se que a violência policial provoca a dispersão da PSR pelo território, dificultando seu cuidado pelas $\mathrm{eCnR}^{18}$. Além disso, as ações de caráter repressivo acarretam em obstáculos no estabelecimento do vínculo, uma vez que as eCnR figuram como representantes do mesmo Estado operador da violência, embora se proponha ao cuidado $^{21}$. É encontrada dificuldade no diálogo e articulação com os setores da segurança pública, da revitalização urbana e da assistência social ${ }^{18,21}$. Essas dificuldades são reforçadas pelo discrepante discurso científico que, embora tenda a afirmar a necessidade de políticas sociais e de saúde para o atendimento das problemáticas que envolvem a PSR, também frequentemente aderem ao discurso do controle que associa de forma acrítica essa população com o uso de drogas e a criminalidade ${ }^{18}$.

Fragilidades nas articulações intersetoriais foram apontadas em dois artigos, problematizando a necessidade de superar as práticas de articulação exclusivamente voltadas a questões de indivíduos em situação de rua visando a construção cotidiana e contínua de ações integradas voltadas para a PSR ${ }^{16,19}$.

Em três pesquisas foram identificadas necessidades formativas dos profissionais que influenciam na articulação das redes. Foi apontada a falta de preparo dos profissionais de elaborarem o cuidado para além das formas pré-concebidas ${ }^{21}$. Também foi evidenciada a necessidade de desenvolver a habilidade de escuta de forma a relacionar necessidades às possibilidades de cuidado presentes nas ofertas da rede de saúde e intersetorial ${ }^{1}$. Por último apareceu o entendimento do princípio da equidade no atendimento de populações vulneráveis, como é o caso da PSR, gerando dúvidas entre profissionais quanto a este ser ou não conflitivo em relação ao princípio da igualdade ${ }^{20}$.

Apenas um estudo apontou dificuldades relativas à dinâmica de vida em situação de rua inerente à população atendida pelo $\mathrm{CnR}$, entendendo que há dificuldades em operar a integralidade do cuidado, uma vez que a PSR comparece aos serviços devido a demandas pontuais, favorecendo o trabalho de caráter ambulatorial. Também há fragilidade na continuidade do cuidado, pois a PSR desloca-se entre territórios, exigindo a flexibilização das eCnR quanto a sua área de abrangência ${ }^{21}$.

\section{POTÊNCIAS}

Todos os estudos apontaram que as eCnR reconhecem a necessidade de articular serviços da Rede de Saúde e Intersetorial para atender as necessidades da PSR. Em especial aqueles cujas metodologias envolveu coleta de dados com usuários do serviço contém relatos de cuidados recebidos por meio do $\mathrm{CnR}$ que implicou no acesso a outros serviços ${ }^{16,19,20}$.

O termo "encaminhamento" foi utilizado por todos os autores para descrever a maneira como o $\mathrm{CnR}$ promove a implicação de outros serviços nos cuidados de usuários, mas 3 deles apontavam práticas que aprofundam o modo de articular a rede: o compartilhamento entre $\mathrm{CnR}$ e outros serviços visando superar a lógica de encaminhamentos formais de modo a promover a coordenação do cuidado ao usuário ${ }^{1,17}$; o trabalho de mapeamento dos serviços presentes na rede e a relação com as necessidades da PSR, 
envolvendo visitas às instituições que visam conhecer seu trabalho e suas rotinas ${ }^{21} ;$ a construção de projetos integrados que envolvam outros serviços da rede, visando superar a articulação exclusivamente para casos individuais, rumo a ações mais amplas ${ }^{1}$.

Quanto ao papel do CnR nas articulações da rede, os estudos foram unânimes em apontar a responsabilidade em promover a ampliação do acesso da PSR aos serviços, mencionando experiências exitosas principalmente no enfrentamento ao preconceito e no reconhecimento da PSR como pessoas de direitos, e a consequente transformação do cotidiano dos serviços para garantir a equidade no atendimento de suas necessidades ${ }^{20,21}$. Cabe destaque a 1 estudo que apontou a potência do trabalho do $\mathrm{CnR}$ como construtor da rede a partir de processos dialógicos com outros serviços, de modo a compreender o trabalho em rede pela coordenação entre serviços ${ }^{1}$.

\section{DISCUSSÃO}

Embora esteja presente nas políticas públicas brasileiras o entendimento de que a atenção à saúde de todo cidadão é responsabilidade de todos os profissionais do SUS, também é prevista a existência de equipes dedicadas ao atendimento de populações específicas como forma de promover a ampliação do acesso, como é o caso das $\mathrm{eCnR}^{5,6}$. Desse modo, é congruente com os achados nos estudos que os desafios e as potências do $\mathrm{CnR}$ no trabalho em rede tenham sido, em sua maioria, relativos ao acesso da PSR aos serviços, tanto pela problematização da burocratização e organização da atenção, quanto pelo enfrentamento do preconceito. Em ambos os casos a promoção do acesso promovida pelo $\mathrm{CnR}$ requer dos profissionais o entendimento das necessidades $\mathrm{e}$ possibilidades de cuidado com a PSR levando em conta suas condições de vida para desvelar as pessoas de direitos que frequentemente são invisibilizadas por práticas engessadas e julgamentos morais.

Esse processo de desconstrução e construção promovido pelo $\mathrm{CnR}$ indica seu papel fluído nas articulações da rede, ora figurando como Equipe de Referência que viabiliza pelo vínculo o acesso e trabalha em favor da coordenação e continuidade do cuidado, ora operando como Apoio Matricial pela ampliação da capacidade de outros serviços quanto aos cuidados da PSR e como dispositivo para a efetivação da equidade no cotidiano do trabalho.

Tendo em vista que o enfrentamento do preconceito com a PSR é previsto como ação educativa abrangente a todos os servidores públicos pela Política Nacional para População em Situação de Rua - PNPSR ${ }^{22}$, cabe questionar quais ações tem sido feitas nesse sentido e com qual grau de eficácia, uma vez que os dados apontam para a atuação do CnR enquanto Apoio Matricial principalmente nessa questão, podendo ter o custo de sobrecarregar esse dispositivo que já enfrenta tantos outros desafios para o cumprimento da atenção à PSR.

Se pretende-se reconhecer o enfrentamento do preconceito com a PSR como alvo de estratégias educativas, a este somam-se outras necessidades formativas de profissionais evidenciadas nos artigos estudados, de modo a compor um eixo orientador da atuação junto à PSR.

O trabalho em rede apresenta, ainda, outras fragilidades relativas à maneira pela qual os serviços se relacionam em suas ações. Nesse contexto, o CnR foi apontado como "construtor da rede", o que constitui potência desse dispositivo pela compreensão da necessidade do trabalho integrado e pelo engajamento na efetivação disso, mas também denuncia que, estando a tarefa de construção concentrada no $\mathrm{CnR}$, existe uma defasagem por parte de profissionais de outros serviços em reconhecer o trabalho em rede como uma necessidade para a atenção integral. A isso está ligada a grande incidência da compreensão desse trabalho pela via do "encaminhamento" e os apontamentos sobre a superação dessa lógica constituir um desafio.

A lógica segundo a qual cada serviço atua ao receber uma demanda e busca atende-la de forma isolada é reflexo da macroestrutura que rege o trabalho nas sociedades capitalistas $^{9,11,13}$. Em razão disso, existe a tendência em compreender as ações de forma setorizada, havendo dificuldades ainda maiores quando a necessidade de construção de ações conjuntas reúne serviços de outros setores. Nesse sentido é importante a compreensão do trabalho em saúde orientado pelo conceito de clínica ampliada, o que teve pouca presença explícita nos estudos analisados, sendo também imprescindível que olhares análogos a esse sejam levados aos profissionais de todos os setores.

\section{CONSIDERAÇÕES FINAIS}

As necessidades da PSR são variadas e exigem esforços de todos os setores públicos para que sejam assegurados seus direitos de cidadania. A atenção a essa população é, portanto, um trabalho complexo que demanda abertura para diálogos e construções entre serviços com a finalidade de compor uma rede que atue de forma coordenada.

Os desafios para a efetivação dessa rede são vários, e perpassam pela transformação de estruturas organizativas que cerceiam direitos ao priorizar a burocracia, o enfrentamento do preconceito que segrega e violenta, a carência de serviços, 
Françoso PA, et al. O cuidado com a população em situação de rua. Rev Ter Ocup Univ São Paulo. 2020 maio/ago.;30(2):124-31.

as contradições entre ações de diferentes atores representantes do Estado, as necessidades formativas dos profissionais.

Nesse cenário, o CnR tem se mostrado dispositivo potente no enfrentamento de algumas dessas problemáticas, mas certamente insuficiente frente a profundidade das fundações macroestruturais que sustentam essas questões na sociedade capitalista.
Assim, este trabalho cumpriu seu objetivo ao evidenciar desafios e potências do trabalho em rede no cotidiano das eCnR, embora seja importante ressaltar a escassez de literatura científica sobre o tema, o que indica os limites desses achados e a necessidade de futuros trabalhos que coloquem em evidência as experiências desse dispositivo de forma a contribuir com a melhoria nos processos de trabalho em rede.

Participação dos autores: Todos os autores contribuíram igualmente para a produção do manuscrito.

\section{REFERÊNCIAS}

1. Engstron EM, Teixeira MB. Equipe "Consultório na Rua" de Manguinhos, Rio de Janeiro, Brasil: práticas de cuidado e promoção da saúde em um território vulnerável. Ciên Saúde Coletiva. 2016;21(6):1839-8. https://doi.org/10.1590/141381232015216.0782016 .

2. Coutinho D, Saback E. O histórico da psiquiatria na Bahia. Gazeta Méd Bahia. 2007;77(2):210-8.

3. Brasil. Ministério da Saúde. Portaria no 4.279, de 30 de dezembro de 2010. Estabelece diretrizes para a organização da Rede de Atenção à Saúde no âmbito do Sistema Único de Saúde (SUS) [citado 20 abr. 2017]. Disponível em: http:// conselho.saude.gov.br/ultimas_noticias/2011/img/07_jan portaria4279_301210.pdf.

4. Brasil. Ministério da Saúde. Portaria $n^{\circ} 1.190$, de 4 de junho de 2009. Institui o Plano Emergencial de Ampliação do Acesso ao Tratamento e Prevenção em Álcool e outras Drogas no Sistema Único de Saúde - SUS (PEAD 2009-2010) e define suas diretrizes gerais, ações e metas [citado 23 jun. 2017]. Disponível em: http://bvsms.saude.gov.br/bvs/saudelegis/ gm/2009/prt1190_04_06_2009.html.

5. Brasil. Ministério da Saúde. Portaria $\mathrm{n}^{\circ} 2.488$, de 21 de outubro de 2011a. Aprova a Política Nacional de Atenção Básica, estabelecendo a revisão de diretrizes e normas para a organização da Atenção Básica, para a Estratégia Saúde da Família (ESF) e o Programa de Agentes Comunitários de Saúde (PACS) [citado 20 abr. 2017]. Disponível em: http://bvsms.saude.gov.br/bvs/saudelegis/gm/2011/ prt2488_21_10_2011.html.

6. Brasil. Ministério da Saúde. Portaria ${ }^{\circ} 122$, de 25 de janeiro de 2011c. Define as diretrizes de organização e funcionamento das Equipes de Consultório na Rua [citado 23 jun. 2017]. Disponível em: http://bvsms.saude.gov.br/bvs/saudelegis/ gm/2012/prt0122_25_01_2012.html.
7. Brasil. Ministério da Saúde. Portaria $n^{\circ} 3.088$, de 23 de dezembro de 2011b. Institui a Rede de Atenção Psicossocial para pessoas com sofrimento ou transtorno mental e com necessidades decorrentes do uso de crack, álcool e outras drogas, no âmbito do Sistema Único de Saúde (SUS) [citado 20 abr. 2017]. Disponível em: http://bvsms.saude.gov.br/ bvs/saudelegis/gm/2011/prt3088_23_12_2011_rep.html.

8. Campos GWS. O anti-Taylor: sobre a invenção de um método para co-governar instituições de saúde produzindo liberdade e compromisso. Cad Saúde Pública. 1998;14(4):863-70. http://dx.doi.org/10.1590/S0102-311X1998000400029.

9. Campos GWS. Equipes de referência e apoio especializado matricial: um ensaio sobre a reorganização do trabalho em saúde. Ciên Saúde Coletiva. 1999;4(2):393-403. http://dx.doi.org/10.1590/S1413-81231999000200013.

10. Campos GWS. Saúde pública e saúde coletiva: campo e núcleo de saberes e práticas. Ciên Saúde Coletiva. 20000;5(2):219-30. http://dx.doi.org/10.1590/S1413-81232000000200002.

11. Campos GWS, Domitti AC. Apoio matricial e equipe de referência: uma metodologia para gestão do trabalho interdisciplinar em saúde. Cad Saúde Pública. 2007;23(2):399-407. http://dx.doi.org/10.1590/ S0102-311X2007000200016.

12. Campos GWS, Cunha GT, Figueiredo MD. Práxis e formação Paideia: apoio e co-gestão em saúde. São Paulo: Hucitec; 2013.

13. Cunha GT, Campos GWS. Apoio matricial e atenção primária em saúde. Saúde Soc. 2011;20(4):961-70. http://dx.doi.org/10.1590/S0104-12902011000400013.

14. Souza MT, Silva MD, Carvalho R. Revisão integrativa: o que é e como fazer. Einstein. 2010;8(1 pt. 1):102-6. doi: 10.1590/s1679-45082010rw1134. 
15. Cooke A, Smith D, Booth A. Beyond PICO: the SPIDER Tool for Qualitative Evidence Synthesis. Qual. Health Res. 2012;22(10):1435-43. https://doi.org/ $10.1177 / 1049732312452938$.

16. Lima HS, Seidl EMFl. Consultório na rua: atenção a pessoas em uso de substâncias psicoativas. Psicol Estudo (Maringá). 2015;20(1):57-69. doi: 10.4025/psicolestud.v20i1.24697.

17. Souza VCA, Pereira AR, Gontijo DT. A experiência no serviço de Consultório de Rua na perspectiva dos profissionais: contribuições para a atenção ao usuário de álcool e outras drogas. Cad Ter Ocup UFSCar. 2014;22(supl. Esp.):37-47. doi: https://doi.org/10.4322/cto.2014.028.

18. Hallais JAS, Barros NF. Consultório na Rua: visibilidades, invisibilidades e hipervisibilidade. Cad Saúde Pública. 2015;31(7):1497-504. https://doi.org/10.1590/0102$311 \mathrm{X} 00143114$.

19. Ferreira CPS, Rozendo CA, Melo GB. Consultório na Rua em uma capital do Nordeste brasileiro: o olhar de pessoas em situação de vulnerabilidade social. Cad Saúde Pública. 2016;32(8):e00070515. https://doi.org/10.1590/0102$311 \times 00070515$.

20. Kami MTM, Larocca LM, Chaves MMN, Piosiadlo LCM, Albuquerque GS. Saberes ideológicos e instrumentais no processo de trabalho no Consultório na Rua. Rev Esc Enferm USP. 2016;50(3):442-96. https://doi.org/10.1590/S0080623420160000400010 .

21. Silva CC, Cruz MM, Vargas EP. Práticas de cuidado e população em situação de rua: o caso do Consultório na Rua. Saúde Debate. 2015;39(n.esp.):246-56. https://doi. org/10.5935/0103-1104.2015S005270.

22. Brasil. Casa Civil. Decreto n ${ }^{0} 7.053$, de 23 de dezembro de 2009 b. Institui Política Nacional para População em Situação de Rua e seu Comitê Intersetorial de Acompanhamento e Monitoramento, e dá outras providências [citado 24 fev. 2018]. Disponível em: http://www.planalto.gov.br/ccivil_03/_ato2007-2010/2009/ decreto/d7053.htm. 